\title{
Spectroscopic studies reveal conformational flexibility of intrinsically unstructured protein HYPK
}

\author{
Swasti Raychaudhuri ${ }^{1,2,3 \#}$, Kamalika Roy Choudhury ${ }^{2 \#}$, Shreoshi Palchoudhuri, ${ }^{1}$, \\ Shradha Chopra ${ }^{1}$, Nitai P. Bhattacharyya ${ }^{2}$, Debashis Mukhopadhyay ${ }^{1^{*}}$

\footnotetext{
${ }^{1}$ Structural Genomics Division, Saha Institute of Nuclear Physics, Kolkata, India;

*Corresponding Author: debashis.mukhopadhyay@saha.ac.in

${ }^{2}$ Crystallography and Molecular Biology Division, Saha Institute of Nuclear Physics, Kolkata, India;

${ }^{3}$ Max Planck Institute of Biochemistry, Department of Cellular Biochemistry, Munich, Germany.
}

Received 3 September 2011; revised 25 October 2011; accepted 7 November 2011.

\begin{abstract}
The chaperone-like huntingtin-interacting protein, HYPK, has unusual biophysical behavior like an intrinsically unstructured protein (IUP). The protein exists as a (pre-) molten globule with $\sim 37 \%$ residual structure and shows compaction in presence of $\mathrm{Ca}^{2+}$. HYPK contains no intrinsic fluorophore other than a single tyrosine and displays an anomalous fluorescence peak at around $340 \mathrm{~nm}$. The anomalous peak is reduced to $303 \mathrm{~nm}$ by the addition of guanidine hydrochloride and at low $\mathrm{pH}$, concomitant with the emission spectrum of L-tyrosine. At high pH the peak is shifted to $\sim 350 \mathrm{~nm}$ with a reduction in intensity. In presence of sodium perchlorate there is no shift in HYPK fluorescence emission peak from $\sim 340 \mathrm{~nm}$ suggesting localization of the lone tyrosine residue in helical regions. In CD experiments, however, a shift in local secondary structure is noticed upon perchlorate treatment. Acrylamide quenching experiments at different $\mathrm{Ca}^{2+}$ concentrations demonstrate that $\mathrm{Ca}^{2+}$ does not alter the accessibility of the tyrosine to acrylamide. In the absence of any tryptophan contamination, these observations validate that, in vitro, HYPK possesses a loosely associated (pre-) molten globule like conformation with the lone tyrosine being situated within an $\alpha$-helix.
\end{abstract}

Keywords: IUP; Huntingtin Yeast-Two Hybrid Protein K; Tyrosine Fluorescence; Conformational Change; Circular Dichroism

\section{INTRODUCTION}

Huntingtin Yeast-two hybrid Protein K (HYPK) is a

${ }^{\#}$ These authors contributed equally to this work. huntingtin-interacting protein that has been shown to reduce aggregation and apoptosis in a Huntington's disease (HD) model [1]. HYPK is shown to have chaperone-like properties $[1,2]$ and is present in a ribosome associated chaperone complex with MPP11 [3]. Our previous study showed that HYPK is an intrinsically unstructured protein (IUP) with a pre-molten globule like conformation [4]. However, under physiological conditions, HYPK is predicted to have $\sim 36.7 \%$ secondary structure [4]. HYPK undergoes a conformational change from a pre-molten globule to a molten globule form, by a calcium-dependent mechanism [4].

It is hypothesized that IUPs interact with multiple partners by attaining different conformations in variable biological contexts $[5,6]$. Disorder-to-order transition is a characteristic of intrinsic disordered protein upon binding with different partners. The flexibility of intrinsic disorder helps different disordered regions to bind to a common binding site on a common partner [7]. It has been reported recently that the anchor residues in the disordered proteins are an important factor in controlling the specific interaction between IDPs and their targets and also play vital role in stabilizing the transient binding complexes [8]. IUPs seem to play important roles in evolution [9] and the binding promiscuity of intrinsically disordered protein plays major roles in the diverse functional properties of unstructured protein.

HYPK has a single aromatic residue $\left(\mathrm{Y}^{49}\right)$, which can be used as a tool to study conformational changes by monitoring intrinsic fluorescence. The unique feature of HYPK fluorescence is its anomalous tyrosine emission at $\sim 330-350 \mathrm{~nm}$ as opposed to normal tyrosine emission at $303 \mathrm{~nm}$ [4]. The origin of this red-shifted tyrosine emission is not fully understood. One possibility is that there is tryptophan contamination in the protein samples [10]. Reports indicate that ionization of the phenolic hydroxyl group of tyrosine leads to tyrosinate formation and red shift in the emission spectrum to $\sim 340 \mathrm{~nm}$ [11]. 
Alternatively, as HYPK is an IUP [4], the tyrosine residue may get trapped in a local conformation and participate in events like excited state proton transfer, leading to a red-shifted fluorescence emission [11]. Functional folding of several proteins is known to depend on the dynamics of their folding pathway intermediates $[12,13]$. These intermediates are often pre-molten globules or molten globules. The folding pathway is frequently influenced by chaperones or ionic conditions [12-14]. Investigating the conformational pliability of intrinsically unstructured HYPK may contribute to understanding the folding of IUPs in the functional context.

In this study we have investigated the conformational properties of HYPK by monitoring intrinsic fluorescence and secondary structure. Our data suggests that the single tyrosine residue in HYPK is located in a conformationally defined $\alpha$-helical region. This study contributes to the structural understanding of HYPK and suggests that structurally-defined regions of IUPs may be functionally important.

\section{MATERIALS AND METHODS}

\subsection{Cloning, Expression and Purification of Recombinant Proteins}

The cloning, expression and purification of full-length HYPK has been previously described [4]. N-terminal (residues 1 - 60) and C-terminal (residues 80 - 129) regions of HYPK were cloned into pET 28a+ (Novagen). HYPK protein fragments were expressed in Escherichia coli BL21 (DE3) cells by IPTG induction (Promega). Cells were resuspended in $50 \mathrm{mM}$ Tris- $\mathrm{Cl} \mathrm{pH} 8.0,300$ $\mathrm{mM} \mathrm{NaCl}, 1 \mathrm{mM}$ PMSF, $2 \mathrm{mM} \beta$-mercaptoethanol and lysed by sonication on ice. The proteins were purified by Ni-NTA affinity chromatography (Qiagen). To dilute the imidazole, samples were dialyzed in $20 \mathrm{mM}$ Tris- $\mathrm{Cl}, \mathrm{pH}$ 7.5. Protein concentrations were determined by Bradford assay.

\subsection{Fluorescence Spectroscopy}

\subsubsection{Fluorescence Spectroscopy with Purified Recombinant HYPK}

Steady-state fluorescence measurements of purified proteins were performed using a Cary Eclipse Fluorescence spectrophotometer (Varian Inc.) and 10-mm path length quartz cuvettes. A protein concentration of $70 \mu \mathrm{M}$ was used. Excitation (at $280 \mathrm{~nm}$ ) and emission (295 $450 \mathrm{~nm}$ ) slits with a $10 \mathrm{~nm}$ band pass were used. Background intensity of the buffer was subtracted from each sample spectrum.

\subsubsection{Effect of pH on HYPK Fluorescence}

To monitor $\mathrm{pH}$ dependent changes, a number of dif- ferent buffers were used. To prepare $\mathrm{pH} 2$ and $\mathrm{pH} 3$ solutions, a $10 \mathrm{mM}$ glycine-based buffer was used $(\mathrm{pH}$ adjusted using $\mathrm{HCl}$ ). For $\mathrm{pH} 4$ and $\mathrm{pH} 5$ solutions, $10 \mathrm{mM}$ acetate was used ( $\mathrm{pH}$ adjusted using acetic acid). The $\mathrm{pH}$ 6 buffer was prepared using $10 \mathrm{mM}$ MES. For pH 7, 8 and 9 solutions, a $10 \mathrm{mM}$ Tris buffer was used $(\mathrm{pH}$ adjusted using $\mathrm{HCl}$ ). For $\mathrm{pH} \mathrm{10,11}$ and 12, a $10 \mathrm{mM}$ glycine solution was used ( $\mathrm{pH}$ adjusted using $\mathrm{NaOH}$ ). 70 $\mu \mathrm{M}$ HYPK was incubated in the different buffers for $2 \mathrm{~h}$ before measurement.

\subsubsection{Effect of Denaturing Agents Like GdmCl or $\mathrm{NaClO}_{4}$ on HYPK Fluorescence}

To study denatured proteins, $70 \mu \mathrm{M}$ HYPK was incubated in $0.5,1,2,3,4,5$ and $6 \mathrm{M}$ guanidium hydrochloride $(\mathrm{GdmCl})$ or in $0.5,1,2,3,4$ and $5 \mathrm{M}$ sodium perchlorate $\left(\mathrm{NaClO}_{4}\right)$ for $2 \mathrm{~h}$.

\subsubsection{Effect of Acrylamide Quenching on HYPK Fluorescence}

Acrylamide quenching studies of HYPK in the presence of $\mathrm{Ca}^{2+}$ or $\mathrm{GdmCl}$ were performed by adding aliquots from a stock solution of acrylamide into a cuvette containing $70 \mu \mathrm{M}$ of protein, previously incubated with either $0,0.1,0.3,0.5,0.7,0.9,1,2,3,4$ and $5 \mathrm{M} \mathrm{CaCl}_{2}$ or $0.5,1,2,3,4,5$ and $6 \mathrm{M} \mathrm{GdmCl}$ for $2 \mathrm{~h}$. Fluorescence quenching data were analyzed using the Stern-Volmer equation for dynamic quenching [15]:

$$
\mathrm{F}_{0} /\left(\mathrm{F}_{0}-\mathrm{F}\right)=\left(1 / \mathrm{f}_{\mathrm{a}} \mathrm{K}_{\mathrm{sv}}[\mathrm{Q}]+1 / \mathrm{f}_{\mathrm{a}}\right)
$$

In this equation $\mathrm{F}_{0}$ and $\mathrm{F}$ are the fluorescence intensities in the absence and presence of quencher, $f_{a}$ is the fraction of accessible fluorophore, $K_{\mathrm{sv}}$ is the dynamic quenching constant (Stern-Volmer constant), and [Q] is the quencher concentration. All data were analyzed using Origin 8.0 and Microsoft Excel.

\subsection{Spectroscopy}

A protein concentration of $70 \mu \mathrm{M}$ was used for $\mathrm{CD}$ experiments. To monitor unfolding proteins were incubated with $0.5,1.0,3.0$ and $5.0 \mathrm{M} \mathrm{NaClO}_{4}$ for $2 \mathrm{~h}$ and far-UV CD spectra were recorded on a CD spectrometer (BioLogic Science Instruments) using a rectangular quartz cell (path length; $0.1 \mathrm{~mm}$ ). Measurements were taken between $190 \mathrm{~nm}$ and $250 \mathrm{~nm}$ at a scan rate of $30 \mathrm{~nm} / \mathrm{min}$. A total of five scans were averaged to obtain each spectrum and they were baseline subtracted for buffer (30 $\mathrm{mM}$ Tris, $\mathrm{pH} 8.0 ; 200 \mathrm{mM} \mathrm{NaCl})$. Percent helicity values were calculated in each case using a previously described equation [16]. The plot for mean residual ellipticity (MRE) versus scan wavelength ( $\mathrm{nm}$ ) was obtained using Origin 8.0. Data smoothing was performed by adjacent averaging with a 25 point window. 


\section{RESULTS}

\subsection{Intrinsic Fluorescence of HYPK}

HYPK has a wide fluorescence emission peak encompassing 330 - $350 \mathrm{~nm}$ compared to L-tyrosine which has a distinct peak at $303 \mathrm{~nm}$ (Figure 1(a)). To rule out the possibility of tryptophan contamination we treated the purified protein with the tryptophan specific reagents $\mathrm{N}-$ bromosuccinidimide and 2-Hydro-5-nitrobenzyl bromide [17]. There was no marked change in absorption and fluorescence spectra of HYPK with these treatments suggesting the absence of contamination (data not shown). Despite the fact that most of the tyrosine residues in proteins have a 10-fold lower fluorescence yield compared to free tyrosine $[18,19]$, the intensity of HYPK fluorescence was about 34 times less than that of L-tyrosine (Figure 1(a)). The absolute quantum yield of intrinsic fluorescence of HYPK was only 0.00414 [18-20]. Hence, the intrinsic fluorescence of HYPK was atypical not only in its emission spectra but also in its fluorescence yield. These anomalies indicated a possible microenvironment of secondary and tertiary structures in the vicinity of the lone tyrosine in HYPK.

\subsection{Accessibility of $Y^{49}$ of HYPK Monitored by Acrylamide Quenching in Varying $\mathrm{Ca}^{2+}$ Concentrations}

Previous work has shown that HYPK undergoes a conformational compaction leading to aggregation in increasing $\mathrm{Ca}^{2+}$ concentrations. The aggregation reaction is concomitant with a reduction in fluorescence intensity [4]. To monitor conformational changes in the region of $\mathrm{Y}^{49}$ we determined the accessibility of the tyrosine residue with increasing concentration of $\mathrm{Ca}^{2+}$ by acrylamide quenching. Considering the low fluorescence quantum yield of HYPK, we used a protein concentration of 70 $\mu \mathrm{M}$ [4]. To rule out any possible effect of residual $\mathrm{Ca}^{2+}$ in experimental buffer, all fluorescence experiments with increasing $\mathrm{Ca}^{2+}$ concentrations were performed after dialyzing the protein against Tris- $\mathrm{Cl}$ buffer $(\mathrm{pH}$ 7) containing $5 \mathrm{mM}$ of EGTA. We determined the fluorescence intensities of HYPK at $340 \mathrm{~nm}$ at variable $\mathrm{Ca}^{2+}$ concentrations and increasing acrylamide concentrations until no aggregation of the protein was observed. The $\mathrm{K}_{\mathrm{sv}}$ (Stern Volmer constant) values were calculated and plotted according to the equation of collisional quenching by Stern-Volmer (Eq.1) (Figure 1(b)) [21]. Only a marginal variation in the Stern-Volmer constant $\left(\mathrm{K}_{\mathrm{SV}}\right)$ values was observed (Figure 1(c)), suggesting no appreciable alterations in the quenching pattern of HYPK intrinsic fluorescence with increasing $\mathrm{Ca}^{2+}$ concentrations. Despite the compaction induced, variation in $\mathrm{Ca}^{2+}$ con- centrations did not affect the accessibility of $\mathrm{Y}^{49}$ of HYPK by acrylamide. The precise effect of $\mathrm{Ca}^{2+}$ was therefore at the global level, altering the loose ensemble of (pre-) molten globules, without significantly influencing the interior formation of the secondary structure.

\subsection{Fluorescence Spectra of HYPK at Increasing $\mathrm{GdmCl}$ Concentrations}

Increasing concentrations of $\mathrm{GdmCl}$ induced a decrease in fluorescence intensity of HYPK at $340 \mathrm{~nm}$ along with the appearance of a tyrosine emission spectrum with a maximum at $303 \mathrm{~nm}$ (Figure 2(a)). However, the emission at $340 \mathrm{~nm}$ still existed even in $6 \mathrm{M} \mathrm{GdmCl}$. We determined the fluorescence emission of HYPK in different $\mathrm{GdmCl}$ concentrations and in the presence of increasing concentrations of acrylamide. As there were two different fluorophores (at 303 and $340 \mathrm{~nm}$ ) with differential accessibilities to acrylamide, the fluorescence quenching data were analyzed by the modified SternVolmer equation (Figures 2(b)-(c)) [15]. The emission at $303 \mathrm{~nm}$ was quenched by acrylamide, but the emission at $340 \mathrm{~nm}$ was not. This result indicated that in the native condition of the protein, tyrosine fluorescence was completely quenched by intra-molecular interactions resulting in emission at $340 \mathrm{~nm}$. Upon GdmCl-induced unfolding, intra-molecular quenching was lost and the tyrosine became accessible to acrylamide. However the fluorophore, emitting at $340 \mathrm{~nm}$, was not accessible to acrylamide as only minimal quenching was observed.

\subsection{Fluorescence Spectra of HYPK in Presence of $\mathrm{NaClO}_{4}$}

$\mathrm{NaClO}_{4}$ is a protein denaturant that differs from guanidine hydrochloride as it leaves helical structures unchanged $[22,23]$. In addition, $\mathrm{NaClO}_{4}$ induces the formation of compact molten globule states by shielding charge repulsion effects within the molecule stabilizing the unfolded state [23]. In our study, treatment with upto $5 \mathrm{M}$ $\mathrm{NaClO}_{4}$ did not result in any shift in HYPK emission maxima; however, there was a reduction in the fluorescence intensity (Figures 3(a)-(b)). As HYPK is an IUP, it is possible that a charge effect results in conformational compaction of the molecule upon addition of sodium perchlorate. This result suggests that the lone tyrosine residue of HYPK is part of an $\alpha$-helical region which the perchlorate ions cannot destabilize.

\subsection{Fluorescence Spectra of HYPK at Different $\mathrm{pH}$}

We measured the effect of $\mathrm{pH}$ on HYPK intrinsic fluorescence. At neutral and slightly alkaline $\mathrm{pH}$, the emission peak was approximately $340 \mathrm{~nm}$ (Figure 3(c)). In 


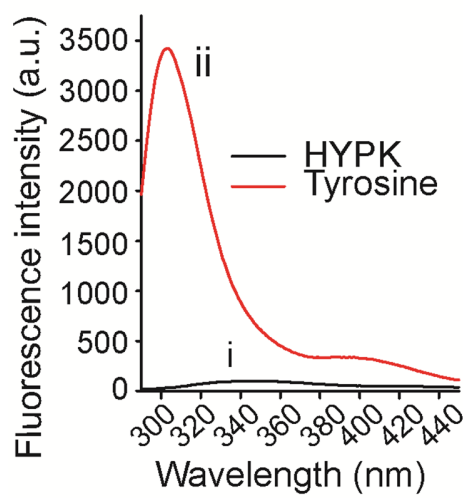

(a)

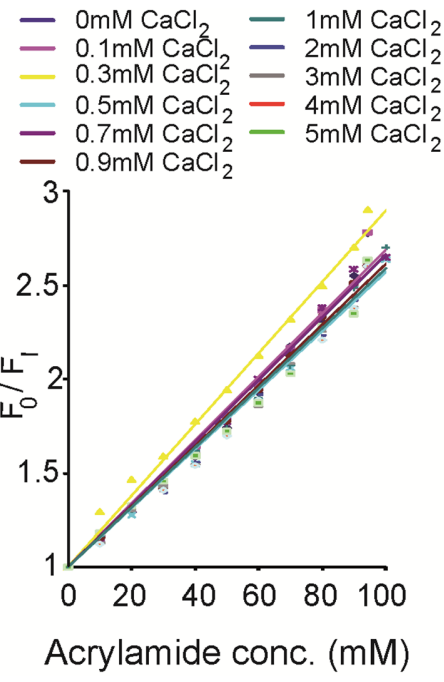

(b)

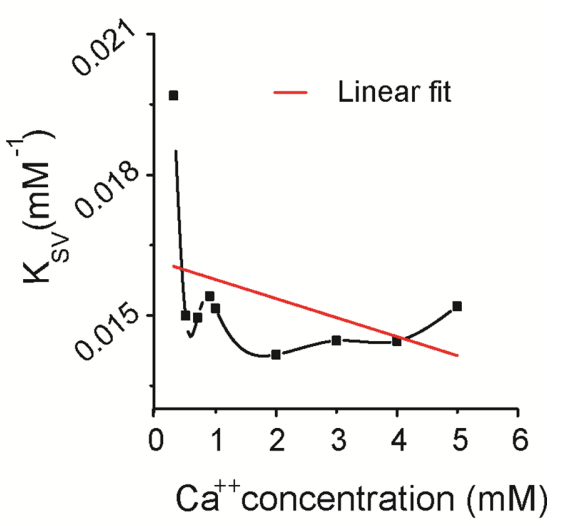

(c)

Figure 1. Anomalous fluorescence of HYPK and quenching in presence of $\mathrm{Ca}^{2+}$. (a) Steady-state fluorescence spectra of purified recombinant HYPK (i), expressed and purified according to Raychaudhuri et al. [1] and dialyzed against $20 \mathrm{mM}$ Tris- $\mathrm{Cl}(\mathrm{pH} 7.5)$ and L-Tyrosine (ii). Samples were taken in equivalent concentrations $\left(2.9 \times 10^{-5} \mathrm{M}\right)$. (b) Stern-Volmer plot of dynamic quenching of intrinsic fluorescence of HYPK. The protein samples $(\sim 70 \mu \mathrm{M}$ each $)$ were dialyzed against $20 \mathrm{mM}$ Tris-Cl pH 7.5 and $5 \mathrm{mM}$ EGTA and were incubated a priori with increasing $\mathrm{Ca}^{2+} \mathrm{concentra-}^{-}$ tions (as described in the figure) for $2 \mathrm{~h}$. Acrylamide quenching studies were performed by adding acrylamide in aliquots from a stock solution directly into cuvette containing the protein. Fluorescence intensities were corrected for dilution effects. Fluorescence quenching data were analyzed using the Stern-Volmer equation for dynamic quenching. (c) $\mathrm{K}_{\mathrm{sv}}$ values, calculated from the Stern-Volmer equation for dynamic quenching (from Figure 1(b)) were plotted against increasing $\mathrm{Ca}^{2+}$ concentrations (as described in figure).

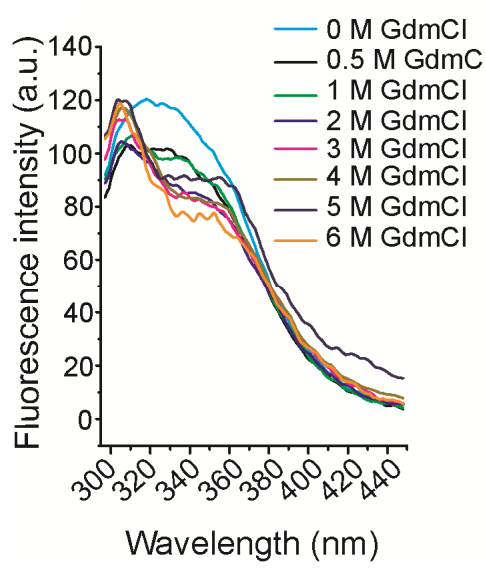

(a)

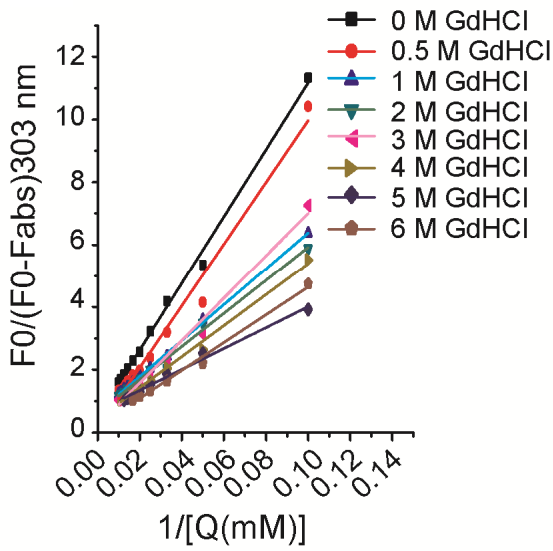

(b)

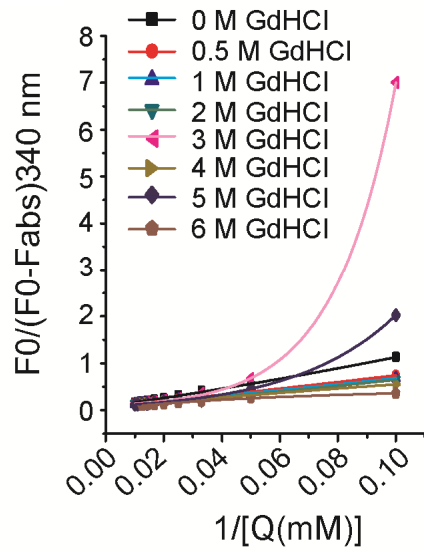

(c)

Figure 2. HYPK fluorescence in presence of GdmCl. (a) Fluorescence spectra of HYPK in presence of increasing guanidine hydrochloride concentrations (as described in figure). Protein samples ( $70 \mu \mathrm{M}$ each) were dialyzed against $20 \mathrm{mM}$ Tris-Cl pH 7.5 and $5 \mathrm{mM}$ EGTA and pre-incubated with $0.5,1,2,3,4,5$ and $6 \mathrm{M}$ guanidine hydrochloride. (b) Stern-Volmer plot of dynamic quenching of fluorescence of HYPK at $303 \mathrm{~nm}$. The protein was incubated a priori with different guanidine hydrochloride concentrations (as described in figure) and quenching studies were performed with increasing acrylamide concentration (as described in figure). (c) Stern-Volmer plot of dynamic quenching of intrinsic fluorescence of HYPK at $340 \mathrm{~nm}$. The protein samples were incubated a priori with different guanidine hydrochloride concentrations (as described in figure) and quenching studies were performed with increasing acrylamide concentration (as described in figure). 


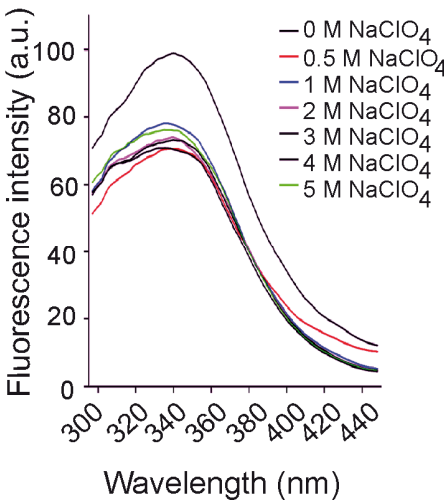

(a)

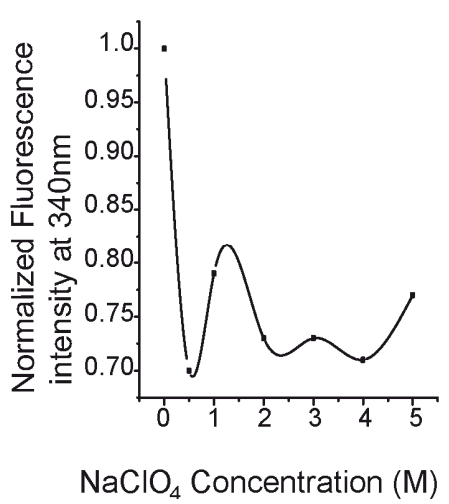

(b)

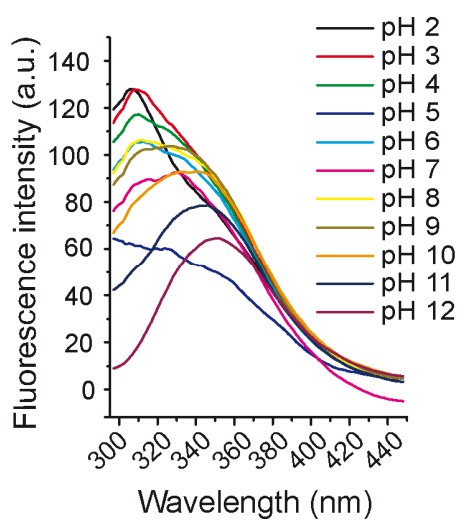

(c)

Figure 3. HYPK fluorescence in presence of $\mathrm{NaClO}_{4}$ and at different $\mathrm{pH}$. (a) Fluorescence spectra of HYPK in presence of increasing sodium perchlorate concentrations. $70 \mu \mathrm{M}$ HYPK was incubated separately with $0.5,1$, 2, 3, 4 and $5 \mathrm{M}$ of sodium perchlorate for $2 \mathrm{~h}$ and fluorescence spectra of the samples were obtained (as described in figure). (b) Normalized fluorescence intensity at $340 \mathrm{~nm}$ were calculated from Figure 3(a) and plotted against increasing $\mathrm{NaClO}_{4}$ concentrations. (c) $\mathrm{pH}$ dependence of intrinsic fluorescence of $\mathrm{HYPK}$. $\sim 70 \mu \mathrm{M} \mathrm{HYPK}$ was incubated separately in different $\mathrm{pH}$ buffers ( $\mathrm{pH} 2$ to $\mathrm{pH} 12)$ for two hours each. After the incubation, fluorescence spectra of the samples were obtained. Note, unusual fluorescence spectra of HYPK at pH 5.

alkaline solutions $(\mathrm{pH} \geq 10)$, the emission peak was red-shifted to $\sim 345-350 \mathrm{~nm}$ with a reduction in intensity. Ionization of $\mathrm{Y}^{49}$ in alkaline solution was similar to that of free tyrosine which has a weak emission at $345 \mathrm{~nm}$ [24]. In contrast, at acidic $\mathrm{pH}$, the peak was shifted to $303 \mathrm{~nm}$. However, due to insoluble aggregation at $\mathrm{pH} 5$ (the pI of HYPK is 5.13) no clear fluorescence data could be recorded. From these data it appears that the effect of $\mathrm{pH}$ on HYPK conformation is similar to $\mathrm{GdmCl}$.

\subsection{Spectroscopy of HYPK with $\mathrm{NaClO}_{4}$}

To obtain further insights we performed CD spectroscopy with full-length HYPK and its N-terminal and Cterminal fragments. HYPK contains very little secondary structure as reported previously [4]. In the presence of low concentrations of $\mathrm{NaClO}_{4}$ the percent $\alpha$-helicity of full-length HYPK was increased (Figure 4(a)). However, at higher $\mathrm{NaClO}_{4}$ concentrations the percent $\alpha$-helicity was again reduced. The N-terminal HYPK (which contains the tyrosine residue) had almost no secondary structure (Figure 4(b)). On the other hand, C-terminal HYPK had substantial $\alpha$-helicity in the presence of increasing concentrations of $\mathrm{NaClO}_{4}$ (Figure 4(c)). These results suggest that with increasing concentrations of $\mathrm{NaClO}_{4}$, the initial increase in helicity in the full-length protein may be due to the C-terminal region which is then gradually compensated by the non-helical N-terminal part.

\section{DISCUSSION}

\subsection{Anomalous HYPK Fluorescence Arise from Excited State Proton Transfer}

Examples of residual structures in IUPs are limited in the literature $[25,26]$. The anomalous fluorescence of HYPK (an IUP), provided us with an opportunity to study its conformational flexibility. Similar proteins with a single tyrosine but no tryptophans are known to have unusual spectroscopic behavior. For example, the excitation spectrum of adrenodoxin has a maxima at $295 \mathrm{~nm}$ [27]. The excitation maximum of HYPK was similar to L-tyrosine at $\sim 280 \mathrm{~nm}$ (data not shown). Nevertheless, the emission spectrum of adrenodoxin resembled HYPK with a peak at around $330-340 \mathrm{~nm}$ but with a higher fluorescence quantum yield [27]. These observations led to the hypothesis that ground state proton transfer contributes to the unusual tyrosine fluorescence of adrenodoxin [27]. Although the emission maximum coincided with the tyrosinate emission peak and the fluorescence quantum yield of HYPK appeared to be compatible with the very low fluorescence yield of tyrosinate [24], the anomalous intrinsic fluorescence of HYPK may be assigned to an intramolecular excited state proton transfer from phenolic hydroxyl to a proton acceptor.

\subsection{Intrinsic Fluorescence of HYPK Solely Contributed by $\mathrm{Y}^{49}$}

In our study, the various denaturants may be disrupting secondary and tertiary structures, therefore affecting the interactions between $\mathrm{Y}^{49}$ and one or more components of the peptide chain. Normalization of the native tyrosine fluorescence to tyrosine fluorescence of HYPK unfolded with $\mathrm{GdmCl}$ and low $\mathrm{pH}$ confirms that the intrinsic fluorescence of native HYPK was solely due to an altered conformational state of $\mathrm{Y}^{49}$. The $\mathrm{pK}_{\mathrm{a}}$ value of tyrosine is 10.3, therefore when HYPK is present in a high $\mathrm{pH}$ solution there is an increased population of ionized and 


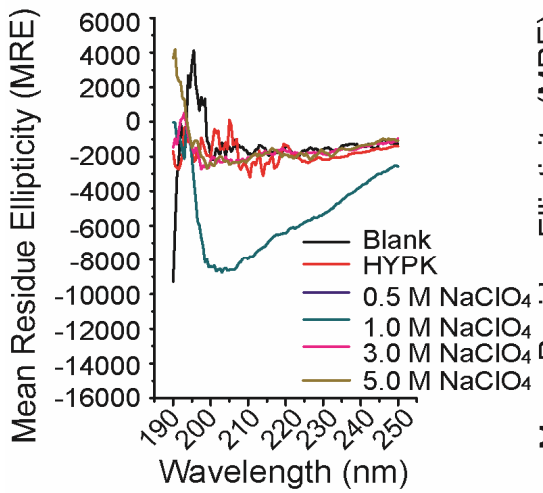

(a)

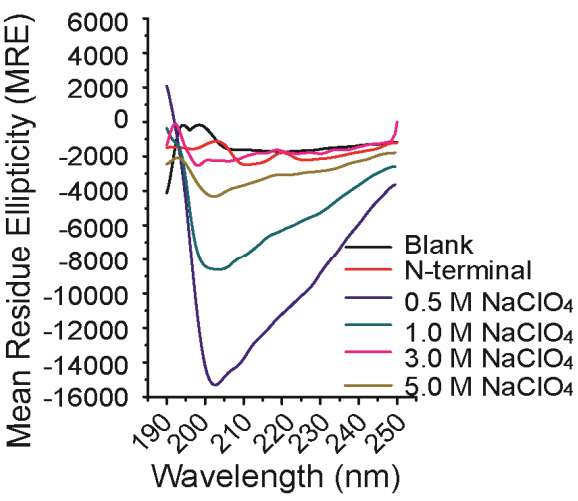

(b)

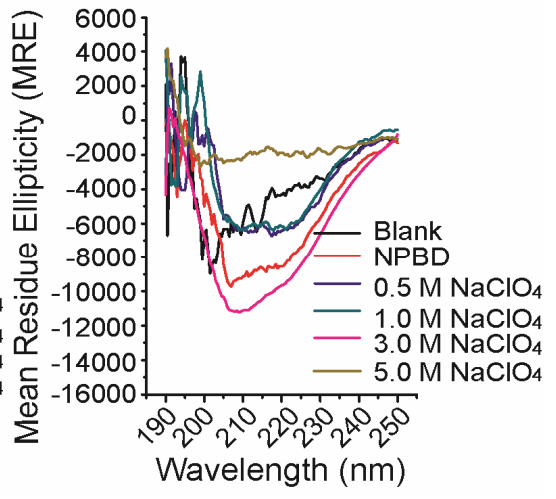

(c)

Figure 4. CD spectral analysis of HYPK in presence of increasing concentrations. $70 \mu \mathrm{M}$ each of full length HYPK (a), N-terminal HYPK, (b) and C-terminal HYPK (c) were incubated separately with a gradient of $0.5 \mathrm{M}, 1.0 \mathrm{M}, 3.0 \mathrm{M}$ and $5.0 \mathrm{M} \mathrm{NaClO}_{4}$ for $2 \mathrm{~h}$ at room temperature and mean residue ellipticity (MRE) were plotted against scan wavelength (nm) range.

stabilized tyrosine. Loss of fluorescence intensity at higher $\mathrm{pH}$ therefore indicates a further compaction of ionized tyrosine possibly through local hydrogen bonding, influenced by neighboring charge(s) or microscopic dielectric constant. $\mathrm{GdmCl}$ had a global unfolding effect, whilst sodium perchlorate failed to perturb the tyrosine environment of HYPK. It is known that sodium perchlorate does not unfold helical structures; therefore our data strongly support the presence of a stable helical structure involving $\mathrm{Y}^{49}$.

\subsection{Dynamic Equilibrium Exists between Tyrosine and Tyrosinate}

Fluorescence quenching by acrylamide is known to be affected by oligomerization which alters the local environment of tyrosine side-chains [26]. However, the acrylamide quenching pattern of HYPK did not change appreciably with increasing $\mathrm{Ca}^{2+}$. In contrast, quenching in the presence of increasing $\mathrm{GdmCl}$ concentrations showed an unfolding of the HYPK structure, causing maximal accessibility of the tyrosine but not the chromophore at $340 \mathrm{~nm}$. This suggests that there is a dynamic equilibrium between two populations of HYPK. The presence of highly dynamic ensembles on interconverting structures is typical of IUPs in solution [28].

\subsection{The $Y^{49}$ Resides in the Transient Local a Helical Structure of HYPK Arising Due to Intramolecular Interaction}

Secondary structure prediction suggests that the lone tyrosine of HYPK is located in a helix (Supplementary Figure 1). Experimentally, however, we did not observe any secondary structure in the N-terminal fragment (which includes $\mathrm{Y}^{49}$ ). CD spectroscopy suggested $\alpha$-helical conformation only in the C-terminal region. Interestingly, the helical content of the full-length protein increased in low concentrations of $\mathrm{NaClO}_{4}$. This might be due to stabilization of the helices which are destabilized again with a further increase in $\mathrm{NaClO}_{4}$ concentration. HYPK contains a number of acidic residues in the local environment of $\mathrm{Y}^{49}$. In a loosely bound molten globule, intramolecular interactions between the phenolic hydroxyl groups of the tyrosine residue and a carboxyl group of the acidic residues may result in formation of transient local structures. Absence of any phenylalanine residues in the protein excluded the possibility of long-range interactions between a phenylalanine and tyrosine. Similar structural compaction of highly charged IUPs in the presence of denaturant has been observed by single molecule FRET [29].

\subsection{The Conformation-Driven Tyrosine Microenvironment Influences the Functional Properties of HYPK}

There is increasing evidence that IUPs are important in biological pathways. Our previous work has confirmed that IUPs are prevalent in neurodegenerative disease networks [30]. We have previously shown that HYPK interacts with Htt and reduces Htt toxicity by modulating the aggregation kinetics by a chaperone-like mechanism. The work of Arnesen et al. suggests that HYPK interacts with NatA and cotranslationally acetylates the $\mathrm{N}$-terminus of $\mathrm{Htt}$, preventing $\mathrm{Htt}$ aggregation and toxicity [31]. Together these studies suggest that HYPK participates in several protein-protein interactions, and may have multiple functionally relevant conformations, an intriguing property of IUPs. This study demonstrates that a conformation-driven microenvironment of the tyrosine residue gives rise to the anomalous spectroscopic properties of HYPK, while the rest of the structure remains highly flexible. This structurally defined region of HYPK 
may have a direct correlation to its function by forming intra-molecular contacts with the flexible part of the protein.

\section{CONCLUSIONS}

In summary, it can be concluded that the anomalous intrinsic fluorescence properties of $\mathrm{Y}^{49}$ residue in HYPK may arise due to excited state proton transfer from phenolic hydroxyl to a proton acceptor present in the local $\alpha$-helical microenvironment around the tyrosine residue. The dynamic equilibrium of this single fluorophor switching between the tyrosine and the tyrosinate forms can contribute to the local compaction of HYPK in presence of $\mathrm{Ca}^{2+}$ and the denaturation of HYPK upon treatment with denaturants like sodium perchlorate or guanidium hydrochloride. This conformation-driven microenvironment change may attribute to various functional properties of many IUPs like HYPK.

\section{ACKNOWLEDGEMENTS}

This work is funded by the SPGHGD project of DAE, Govt. of India. We are grateful to Prof. A. Chakrabarti, Prof. P. K. Sengupta, Mrs. B. Chakraborty, Mrs. S. Chakraborty and Mr. B. P. Pahari for helpful discussion. Dr. Amy Robertson is sincerely thanked for checking the language.

\section{REFERENCES}

[1] Raychaudhuri, S., Sinha, M., Mukhopadhyay, D. and Bhattacharyya, N.P. (2008) HYPK, a Huntingtin interacting protein, reduces aggregates and apoptosis induced by $\mathrm{N}$-terminal Huntingtin with 40 glutamines in Neuro2a cells and exhibits chaperone-like activity. Human Molecular Genetics, 17, 240-255. doi:10.1093/hmg/ddm301

[2] Szolajska, E. and Chroboczek, J. (2011) Faithful chaperones. Cellular and Molecular Life Sciences, 68, 33073322. doi:10.1007/s00018-011-0740-4

[3] Otto, H., Conz, C., Maier, P., Wolfle, T., Suzuki, C.K., Jeno, P., Rucknagel, P., Stahl, J. and Rospert, S. (2005) The chaperones MPP11 and Hsp70L1 form the mammalian ribosome-associated complex. Proceedings of the $\mathrm{Na}$ tional Academy of Sciences of the United States of America, 102, 10064-10069. doi:10.1073/pnas.0504400102

[4] Raychaudhuri, S., Majumder, P., Sarkar, S., Giri, K., Mukhopadhyay, D. and Bhattacharyya, N.P. (2008) Huntingtin interacting protein HYPK is intrinsically unstructured. Proteins, 71, 1686-1698. doi:10.1002/prot.21856

[5] Tompa, P. (2002) Intrinsically unstructured proteins. Trends in Biochemical Sciences, 27, 527-533. doi:10.1016/S0968-0004(02)02169-2

[6] Tompa, P., Szasz, C. and Buday, L. (2005) Structural disorder throws new light on moonlighting. Trends in Biochemical Sciences, 30, 484-489.

doi:10.1016/j.tibs.2005.07.008

[7] Dunker, A.K., Oldfield, C.J., Meng, J., Romero, P., Yang,
J.Y., Chen, J.W., Vacic, V., Obradovic, Z. and Uversky, V.N. (2008) The unfoldomics decade: An update on intrinsically disordered proteins. BMC Genomics, $\mathbf{9}, \mathrm{S} 1$. doi:10.1186/1471-2164-9-S2-S1

[8] Huang, Y. and Liu, Z. (2011) Anchoring intrinsically disordered proteins to multiple targets: Lessons from $\mathrm{N}$-terminus of the p53 protein. International Journal of Molecular Sciences, 12, 1410-1430. doi:10.3390/ijms12021410

[9] Dunker, A.K. and Kriwacki, R.W. (2011) The orderly chaos of proteins. Scientific American, 304, 68-73. doi:10.1038/scientificamerican0411-68

[10] Ross, J.B.A., Laws, W.R., Rousslang, K.W. and Wyssbrod, H.R. (1992) Topics in fluorescence spectroscopy: Biochemical applications. In: Lakowicz, J.R., Ed., Biochemical Applications, Plenum Press, New York, 49-50.

[11] Ross, J.B.A., Laws, W.R., Rousslang, K.W. and Wyssbrod, H.R. (1992) Topics in fluorescence spectroscopy: Biochemical applications. In: Lakowicz, J.R., Ed., Biochemical Applications, Plenum Press, New York, 2-5.

[12] Myers, J.K. and Oas, T.G. (2002) Mechanism of fast protein folding. Annual Review of Biochemistry, 71, 783-815. doi:10.1146/annurev.biochem.71.110601.135346

[13] Flynn, G.C., Beckers, C.J., Baase, W.A. and Dahlquist, F.W. (1993) Individual subunits of bacterial luciferase are molten globules and interact with molecular chaperones. Proceedings of the National Academy of Sciences of the United States of America, 90, 10826-10830. doi:10.1073/pnas.90.22.10826

[14] Arai, M. and Kuwajima, K. (2000) Role of the molten globule state in protein folding. Advances in Protein Chemistry, 53, 209-282. doi:10.1016/S0065-3233(00)53005-8

[15] Lakowicz, J., Gryczynski, I., Gryczynski, Z., Tolosa, L., Randers-Eichhorn, L. and Rao, G. (1999) Principles of fluorescence spectroscopy. Kluwer Academic/Plenum Publishers, New York.

[16] Lucas, H.R. and Lee, J.C. (2011) Copper (II) enhances membrane-bound alpha-synuclein helix formation. Metallomics, 3, 280-283. doi:10.1039/c0mt00088d

[17] Privat, J.P., Lotan, R., Bouchard, P., Sharon, N. and Monsigny, M. (1976) Chemical modification of the tryptophan residues of wheat-germ agglutinin. Effect on fluorescence and saccharide-binding properties. European Journal of Biochemistry, 68, 563-572. doi:10.1111/j.1432-1033.1976.tb10844.x

[18] Ross, J.B.A., Laws, W.R., Rousslang, K.W. and Wyssbrod, H.R. (1992) Topics in fluorescence spectroscopy: Biochemical applications. In: Lakowicz, J.R., Ed., Biochemical Applications, Plenum Press, New York, 44-45.

[19] Chen, R.F. and Kernohan, J.C. (1967) Combination of bovine carbonic anhydrase with a fluorescent sulfonamide. Journal of Biological Chemistry, 242, 5813-5823.

[20] Wong, M., Thomas, J.K. and Graetzel, M. (1976) Fluorescence probing of inverted micelles. The state of solubilized water clusters in alkane/diisooctyl sulfosuccinate (aerosol OT) solution. Journal of the American Chemical Society, 98, 2391-2397.

[21] Eftink, M.R. (1991) Topics in fluorescence spectroscopy: Principles. In: Lakowicz, J.R. Ed., Principles, Plenum Press, New York, 55-56. 
[22] Maruyama, S., Kuwajima, K., Nitta, K. and Sugai, S. (1977) Thermodynamic characterization of partially denatured states in the denaturation process of bovine alpha-lactal-bumin by inorganic denaturants. Biochimica et Biophysica Acta, 494, 343-353.

[23] Asciutto, E.K., General, I.J., Xiong, K., Asher, S.A. and Madura, J.D. (2010) Sodium perchlorate effects on the helical stability of a mainly alanine peptide. Biophysical Journal, 98, 186-196. doi:10.1016/j.bpj.2009.10.013

[24] Cornog Jr., J.L. and Adams, W.R. (1963) The fluorescence of tyrosine in alkaline solution. Biochimica et Biophysica Acta, 66, 356-365. doi:10.1016/0006-3002(63)91204-6

[25] Uren, A., Tcherkasskaya, O. and Toretsky, J.A. (2004) Recombinant EWS-FLI1 oncoprotein activates transcription. Biochemistry, 43, 13579-13589. doi:10.1021/bi048776q

[26] Permyakov, S.E., Millett, I.S., Doniach, S., Permyakov, E.A. and Uversky, V.N. (2003) Natively unfolded C-terminal domain of caldesmon remains substantially unstructured after the effective binding to calmodulin. Proteins, 53, 855-862. doi:10.1002/prot.10481

[27] Lim, B.T. and Kimura, T. (1980) Conformation-associated anomalous tyrosine fluorescence of adrenodoxin. Journal of Biological Chemistry, 255, 2440-2444.
[28] Xie, H., Vucetic, S., Iakoucheva, L.M., Oldfield, C.J., Dunker, A.K., Uversky, V.N. and Obradovic, Z. (2007) Functional anthology of intrinsic disorder. 1. Biological processes and functions of proteins with long disordered regions. Journal of Proteome Research, 6, 1882-1898. doi:10.1021/pr060392u

[29] Muller-Spath, S., Soranno, A., Hirschfeld, V., Hofmann, H., Ruegger, S., Reymond, L., Nettels, D. and Schuler, B. (2010) From the cover: Charge interactions can dominate the dimensions of intrinsically disordered proteins. Proceedings of the National Academy of Sciences of the United States of America, 107, 14609-14614. doi:10.1073/pnas.1001743107

[30] Raychaudhuri, S., Dey, S., Bhattacharyya, N.P. and Mukhopadhyay, D. (2009) The role of intrinsically unstructured proteins in neurodegenerative diseases. PLoS One, 4, e5566. doi:10.1371/journal.pone.0005566

[31] Arnesen, T., Starheim, K.K., Van Damme, P., Evjenth, R., Dinh, H., Betts, M.J., Ryningen, A., Vandekerckhove, J., Gevaert, K. and Anderson, D. (2010) The chaperone-like protein HYPK acts together with NatA in cotranslational N-terminal acetylation and prevention of Huntingtin aggregation. Molecular and Cellular Biology, 30, 18981909. doi:10.1128/MCB.01199-09 
Secondary structure of HYPK (predicted)

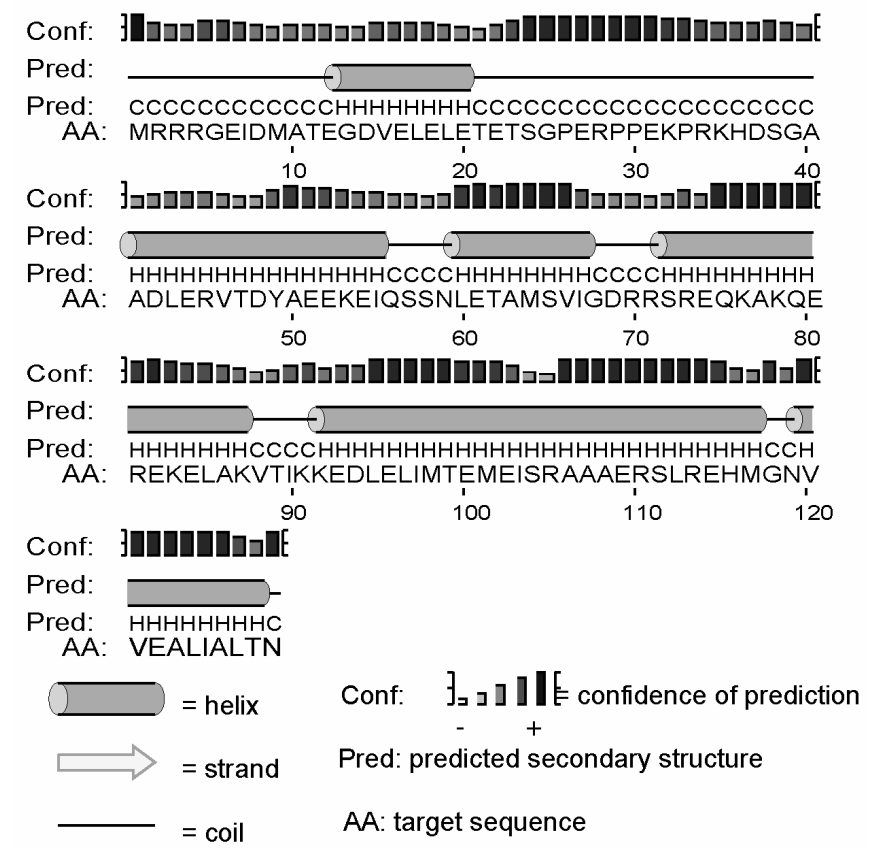

(a)

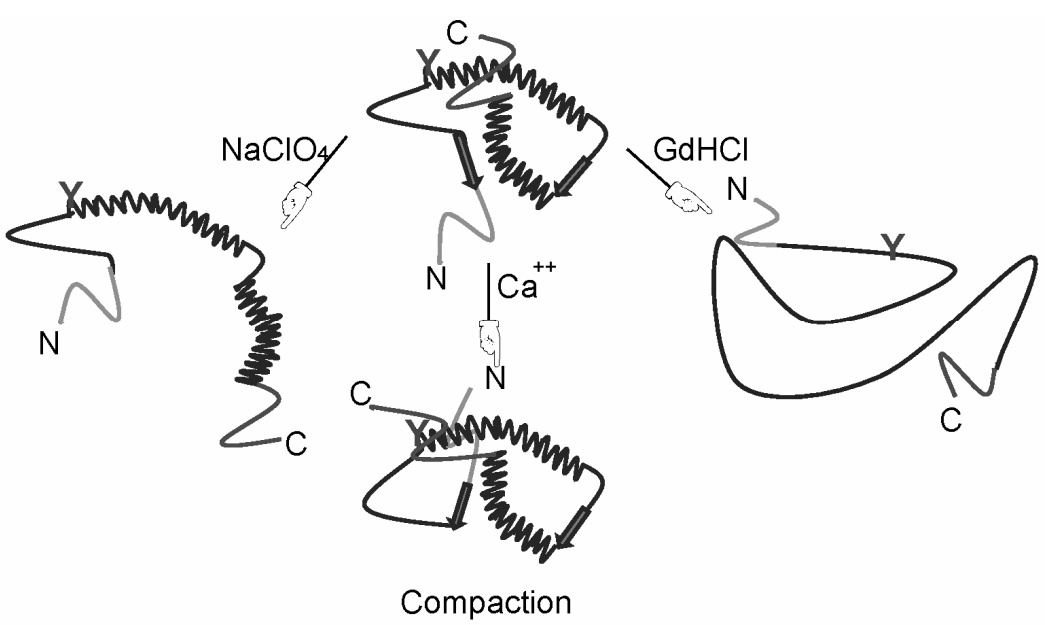

(b)

Supplementary Figure 1. Secondary structure of HYPK. (a) Secondary structure of HYPK as predicted by PSIPRED [1]; (b) Schematic diagram showing conformational alteration of HYPK at different ionic ambience. Possible location of tyrosine residue in the protein at different conditions is indicated in green. 